\title{
Band application of flue gas desulfurization gypsum improves sodic soils amelioration
}

\author{
Wenchao Zhang ${ }^{1}$, Wenxin Zhang ${ }^{1}$, Shujuan $\mathrm{Wang}^{2}$, Jia Liu ${ }^{1}$, Yan $\mathrm{Li}^{1}$, Yuqun Zhuo ${ }^{1}$, Lizhen \\ $\mathrm{Xu}^{1}$, and Yonggan Zhao ${ }^{1}$ \\ ${ }^{1}$ Tsinghua University \\ ${ }^{2}$ Tsinghua Univ
}

October 13, 2020

\begin{abstract}
Blending FGD gypsum with surface sodic soil is a universally recognized method for the rapid amelioration of sodic soils; however, there are few reports on whether other application methods (band application) will reclaim sodic soil. Three FGD gypsum application methods, single-band application, dual-band application and blending, were carried out using sodic soil in soil bins to investigate the effects of application method on the wetting front, major cation concentrations and ESP during the process of water infiltration and in the soil profile after infiltration. The results showed that the wetting fronts in the band treatments were denser in the horizontal direction than in the vertical direction, but the blend treatment only had vertical migration. There was an exponential relationship between time and depth of penetration. The orders of desalting capacity were blend treatment, dual-band treatment and single-band treatment for the same volume of outlet water. Na+ was the primary ion in the leachate. The main channel in the band treatments was concentrated below the application site of FGD gypsum. The dual-band treatment significantly decreased the soil ESP of the whole soil bin, while the single-band treatment only effectively reclaimed half of the soil. In the blend treatment, the ESP was $21.32 \%$ and $34.66 \%$ at depths of $30-35 \mathrm{~cm}$ and $35-40 \mathrm{~cm}$ and was close to zero at a depth of 0-30 cm. Compared with blend treatment, band treatments have the advantage of long-term amelioration of local sodic soil, and the performance is mainly affected by the Ca2+ pathway.
\end{abstract}

\section{Hosted file}

Manuscript------\begin\{CJK\}\{UTF8\}\{gbsn\}. \end\{CJK\}\selectlanguage\{english\}pdf available } at https://authorea.com/users/366891/articles/486503-band-application-of-flue-gasdesulfurization-gypsum-improves-sodic-soils-amelioration 